\title{
Nuevos medios: usos comunicativos de los adoles- centes. Perspectivas desde los nativos digitales ${ }^{1}$
}

Recibido: 3 de junio de 2013

\author{
José Carlos SEndín GuTIÉRREZ \\ Universidad Rey Juan Carlos \\ josecarlos.sendin@urjc.es \\ Carmen GaOna Pisonero \\ Universidad Rey Juan Carlos \\ carmen.gaona@urjc.es
Antonio GARCÍA JIMÉNEZ
Universidad Rey Juan Carlos
antonio.garcia@urjc.es

Aceptado: 2 de diciembre de 2014

\begin{abstract}
Resumen
Este trabajo presenta los resultados de un proyecto de investigación de ámbito estatal sobre hábitos y dinámicas de los adolescentes en Internet. Concretamente se presentan los resultados derivados de diferentes grupos de discusión tomando en consideración el uso. Esta faceta cualitativa de la investigación también está conectada con la cuantitativa, a través de una encuesta aplicada a nivel estatal. Además de confirmar la tendencia internacional y nacional del acceso a Internet a menor edad, se refuerza el comportamiento multitarea de los adolescentes. La experiencia digital se individualiza, al tiempo que la presencia en redes sociales es generalizada. Finalmente, se constata una percepción diferente de los riesgos en Internet, expresada en una reformulación del concepto de privacidad que apunta tendencias de investigación futuras en un ámbito objeto de creciente interés académico.
\end{abstract}

Palabras clave: Adolescentes, Internet, redes sociales, usos, relaciones familiares.

\section{New media: Uses and communication by adolescents. Prospects from digital natives}

\begin{abstract}
This paper presents the results of a research project on the state level on uses and dynamics driving habits of teenagers on the Internet. Specifically, results are derived from different discussion groups considering use. This qualitative aspect of this research is also connected with the quantitative, through a statewide survey. In addition to confirming the international and national trend of Internet access at a younger age, it reinforces the multitasking behavior of adolescents. The digital experience is individualized, while the social networking presence is widespread. Finally, a different perception of the risks on the Internet is noted, expressed in a reformulation of the concept of privacy, that point out future research trends in a field with a growing academic interest.
\end{abstract}

Keywords: Adolescents, Internet, social networks, uses, family relations.

1 Este artículo forma parte de la investigación emprendida en el Proyecto Nacional de I+D+I "Análisis de uso y consumo de medios y redes sociales en Internet entre los adolescentes españoles. Características y prácticas de riesgo" (CSO2009-09577), Ministerio de Ciencia e Innovación. Secretaría de Estado de Investigación. Dirección General de Investigación y Gestión del Plan Nacional de I+D+I. Miembros del proyecto: Antonio García Jiménez, Ma del Carmen Arellano Pardo, Beatriz Catalina García, Ma Edisa Mondelo González, Carmen Pérez Pais, $\mathrm{M}^{\mathrm{a}}$ del Carmen Gaona Pisonero, $\mathrm{M}^{\mathrm{a}}$ Cruz López de Ayala López, Flávia Gomes Franco e Silva, Esther Martínez Pastor, Ma Pilar Beltrán Orenés, Manuel Montes Vozmediano, Rosa San Segundo Manuel, José Carlos Sendín. 


\section{Referencia normalizada}

SENDÍN GUTIÉRREZ, José Carlos; GAONA PISONERO, Carmen; y GARCÍA JIMÉNEZ, Antonio (2014): "Nuevos medios: usos comunicativos de los adolescentes. Perspectivas desde los nativos digitales". Estudios sobre el Mensaje Periodístico. Vol. 20, Núm. 1 (enero-junio), págs.: 265-280. Madrid, Servicio de Publicaciones de la Universidad Complutense.

Sumario: 1. Introducción. 2. Estado de la cuestión. 3. Objetivo y preguntas de investigación, 4. Metodología. 5. Resultados; 5.1. Usos; 5.1.1. Uso del ordenador. Grupo 12-14 años; 5.1.2. Uso del ordenador. Grupo 15-17 años; 5.1.3. Uso de la televisión. Grupo 12-14 años; 5.1.4. Uso de Internet. Grupo 12-14 años; 5.2. Relaciones sociales en Internet. Grupo 15-17 años; 5.3. Padres y familia; 5.3.1. Padres y familia. Grupo 15-17 años. 6. Conclusiones. 7. Referencias bibliográficas.

\section{Introducción}

En este trabajo se presentan los resultados obtenidos a partir de diferentes grupos de discusión cuya aplicación ya fue testada en un estudio anterior, centrado solo en la Comunidad de Madrid. Los hábitos y prácticas de los menores en Internet constituye un objeto de estudio con una creciente atención por parte de la academia, por ello esta investigación complementa la importante presencia de trabajos cuantitativos, incorporando el análisis de las percepciones de los menores y adolescentes a partir de sus narrativas y discursos, que ayudan a entender los significados que dan los menores a sus prácticas comunicativas y digitales, y ofrece tendencias para la investigación futura.

\section{Estado de la cuestión}

Este trabajo se sitúa en la intersección de teorías y metodologías utilizadas por investigaciones similares que se han centrado en la relación entre los jóvenes y el Internet. De hecho, nuestro estudio está en consonancia con el trabajo realizado en el marco de EU Kids Online, que ha dado lugar a una enorme bibliografía (Livingstone, 2008; Livingstone \& Brake, 2010; Livingstone et al, 2011, entre otros), así como otras investigaciones en la misma línea (Valcke et al., 2011). A pesar de que los menores e Internet han sido objeto de un incremento considerable del esfuerzo investigador en los últimos años, parece que todavía los marcos existentes no acaban de proporcionar una imagen completa de los factores que influyen en la selección de tecnologías de la información y la comunicación (TIC) por parte de los adolescentes.

En primer lugar, nos centramos en la percepción sobre los usos que los adolescentes hacen de Internet y de las redes sociales. La mayoría de las investigaciones realizadas en nuestro país desde 2008 coinciden en señalar que la práctica totalidad de los jóvenes se conecta a Internet. Hay una clara tendencia hacia la universalización entre los adolescentes y jóvenes, grupos donde el 87,1\% (10-15 años) y 95\% (16-24 años) ya son usuarios, mientras que el $88,5 \%$ de los jóvenes internautas de entre 16 y 24 años participan en las redes sociales. (INE, 2012). La falta de medios, o un bajo estatus socioeconómico son los motivos principales argumentados por los jóvenes que no acceden a Internet. Las diferencias de género son relevantes, puesto que se detecta una navegación sensiblemente mayor entre las chicas que entre los chicos, pero en la mayoría de los estudios no superan el 60-40\%, a excepción del informe de Eu Kids Online que registra un $76 \%$ de uso entre las chicas frente un $59 \%$ entre los chicos. (García et al. 2012). 
En la mayor parte de la literatura científica se constata que cuando se hace referencia al lugar de conexión, un importante porcentaje de los menores accede a Internet desde su casa (en segundo lugar, aparece el colegio), siendo lo más habitual acceder desde su habitación (49\% frente a cualquier otra ubicación de la casa, 38\%). Por otra parte, la mayoría se conecta desde un PC compartido (59\%). Y si bien se ha incrementado el acceso a Internet desde un dispositivo móvil, es palpable que en este aspecto tiene influencia el estatus socioeconómico de la familia (Garmendia et al., 2011).

Por lo que respecta al uso de redes sociales, el trabajo de Sánchez-Navarro y Aranda (2011) destaca que los principales usos de las redes sociales son hablar con amigos y saber lo que hacen, opinar, buscar información y solucionar dudas de los estudios. De igual modo, Ochaita, Espinosa y Gutiérrez (2011), apuntan que mientras un $29 \%$ ve la televisión en soledad, esta cifra alcanza el $76,3 \%$ cuando se incorporan al ciberespacio. En este sentido, las actividades que parecen estar más afectadas negativamente por la navegación en Internet son: leer (con un 21,1\%), hacer deporte $(13,6 \%)$, y el estudio $(10,8 \%)$. De igual modo, son pocos los adolescentes que reconocen ser objeto de alguna forma de control parental.

Asimismo, se constata que los niños y adolescentes de 10 a 18 años acceden a Tuenti en un 60\% (Río, Sádaba y Bringué, 2010: 124). Las cifras de preferencia por Tuenti son más altas si se observan otros estudios: un $80 \%$ de adolescentes españoles se decanta por esta red social (Sánchez Burón y Fernández Martín, 2010: 11). En cualquier caso, la elección de Tuenti predomina sobre las demás y, en segundo lugar, se sitúa Facebook.

La investigación internacional señala características similares a las ya identificadas y algunas diferenciales. Aparece el elevado porcentaje de uso de Internet en los adolescentes y la cada vez menor edad de acceso, combinadas con el papel protagonista del móvil (Purcell, 2011). Varios autores han detectado en diferentes países la tendencia al uso intensivo de Internet, expresión preferida en lugar de otras, como uso adictivo, que implica valoración negativa y sobre la que no existe consenso generalizado, (Lenhart, A. \& Madden, M., 2007; Lenhart, Purcell, K., Smith, A., \& Zickuhr, K., 2010). Por otro lado, Cheung et al. (2011) han detectado que entre el grupo de edad entre 19 y 23 años, los roles implícitos y explícitos del grupo influencian el uso de Facebook, pues otros investigadores detectaron que esos roles de grupo eran adoptados como patrones de conducta (Walsh et al., 2009). Además, frente a la idea generalizada sobre que los adolescentes tienen poca información y que usan las redes de manera poco segura, diferentes estudios demuestran que los adolescentes, por el contrario, conocen los riesgos potenciales de Internet para su seguridad y privacidad y toman precauciones al respecto (Patchin \& Hinduja, 2010).

En segundo lugar, destacamos la literatura que se centra en las relaciones digitales entres los adolescentes. Y el primer aspecto que destaca el de la amistad. En lo que respecta al concepto de amistad, Foucault and Markov (2009) definen dos categorías: amistad instrumental y amistad afectiva. La primera es limitada en profundidad y alcance, mientras que la afectiva es resultado de lazos más profundos no motivados por la búsqueda de objetivos específicos. En otros textos se aprecia un cambio en este sentido, ya que en estudios iniciales (Nie, 2001; Mesch, 2001), se en- 
tendía que el ciberespacio favorecía formas de relación online con extraños; sin embargo, en los estudios más recientes se comprueba que estas relaciones se orientan a mantener los lazos de amistad (Boyd, 2007; Gross, 2004; Livingstone \& Brake, 2010; Mesch \& Talmud, 2007; Valkenburg \& Peter, 2009). Y, además, otros estudios apuntan que estas formas de comunicación tienen efectos positivos en la amistad (Valkenburg \& Peter, 2009, 2011). Otros investigadores se han centrado en la naturaleza superficial y simple de los mensajes, así como en la importancia de las herramientas para estar constantemente conectados a amigos y conocidos (Ito et al., 2009; Subrahmanyam \& Smahel, 2011).

En otros trabajos se ha analizado la relación entre la conectividad, el sentimiento de pertenencia y la identidad social de los adolescentes. De acuerdo con Valkenburg y Peter (2009), conectividad se define como la relación entre los adolescentes en el ciberespacio, que es su medio. En esta línea, se constata que estar online ayuda a mantener la relación offline (Subrahmanyam, Reich, Waechter, \& Espinoza, 2008; Ellison et al, 2007), especialmente en las redes sociales como Facebook (Barkhuus \& Tashiro, 2010). Walsh, White, and Young (2009) relacionan el sentimiento de pertenencia con la capacidad de comprensión, control, exaltación del yo, así como el proceso de construcción de la propia identidad.

En tercer lugar, estudios como el de Duerager \& Livingstone (2012) analizan la influencia del contexto familiar en el uso de Internet por los adolescentes. En particular, las relaciones familiares insatisfactorias (Liu et al., 2012; Viñas et al., 2002; Lam et al., 2009), la comunicación familiar (Liu et al., 2012; Park et al., 2008) y cómo el uso intensivo y problemático de Internet se relaciona con los conflictos entre padres e hijos (Yen et al., 2007).

\section{Objetivo y preguntas de investigación}

En este trabajo nos planteamos como objetivo acercarnos a los hábitos y dinámicas de los adolescentes en Internet, a través de sus propios relatos. Este objetivo se articula en tres preguntas de investigación. En primer lugar, cómo acceden y qué usos llevan a cabo los adolescentes en Internet. En segundo lugar, qué cambios se ha producido, en el caso de que haya sido así, en la visión de los adolescentes sobre las relaciones sociales y emocionales, a consecuencia del uso diario de Internet. Y, por último, cuál es la percepción de los adolescentes sobre el papel de los padres y la familia en relación con Internet.

\section{Metodología}

El análisis aquí presentado forma parte de un estudio más amplio ${ }^{2}$ que combina metodología cuantitativa y cualitativa. El diseño de la muestra, el tamaño y su distribución se calcularon a partir de los datos de matriculación del último año disponible, fa-

2 Proyecto Nacional de I+D+I "Análisis de uso y consumo de medios y redes sociales en Internet entre los adolescentes españoles. Características y prácticas de riesgo" (CSO200909577), Ministerio de Ciencia e Innovación. Secretaría de Estado de Investigación. Dirección General de Investigación y Gestión del Plan Nacional de I+D+I. 
cilitados por el servicio de estadística educativa del Ministerio de Educación (20092010).

La unidad de observación de la parte cualitativa objeto de este trabajo, se corresponde con diferentes centros educativos, con vistas a aplicar la técnica de grupos de discusión. Sobre la estructura de los grupos de discusión, prevalecen en su perfil muestral las variables de centro con interés educativo en las TICs, el género, la edad, el grupo socioeconómico y alto consumo digital. Se trabajó con grupos mixtos separados de pre-adolescentes y de adolescentes, considerados de forma operativa como individuos de 12-14 años (niños) y de 15-17 (adolescentes). Se seleccionaron seis institutos públicos: Andalucía (1), Cataluña (2), Madrid (2), Murcia (1), y dos centros concertados: Galicia (1) y Aragón (1).

Una vez seleccionados los centros, se solicitaron las correspondientes autorizaciones paternas para participar en el proyecto y se estableció contacto con las direcciones de los diferentes centros, que se ocuparon de la selección de los menores con las indicaciones relativas a una elección no condicionada de los alumnos. Los grupos de discusión, que tuvieron una duración de una hora de media aproximadamente y contaron con una media de seis menores, fueron dirigidos por un moderador que se encargó de organizar cada sesión, con un conjunto de puntos previamente establecidos al hilo tanto de los usos como de las diferentes dinámicas en Internet. La tarea de los moderadores era potenciar el debate en cada grupo con flexibilidad, y reconducirlo en aquellas ocasiones en las que la discusión se adentraba en temas ajenos a los objetivos planteados.

Todos los grupos de discusión fueron grabados y transcritos en su totalidad. Tras repasar y corregir los textos de las transcripciones se procedió a una primera segmentación a partir de toda la información recogida, siempre en función de la importancia semántica establecida por los objetivos de nuestra investigación. En esta tarea se empleó el programa ATLAS.ti.

Se codificó tomando en consideración quién hablaba, su género y edad. A continuación, se realizó el correspondiente procesamiento automático generándose 14 códigos semánticos: 9 vinculados a los usos y 5 a los riegos y a las formas de control, tras ser testados por los miembros del grupo de investigación. En un porcentaje muy alto, se correspondían con las categorías extraídas a partir de cinco grupos de discusión realizados en el mencionado proyecto de investigación anterior, limitado a los adolescentes en la Comunidad de Madrid de España (García, 2010). Por otro lado, estas categorías se adaptaban a los objetivos específicos establecidos en el proyecto, y se compararon con dos grupos focales iniciales con el fin de establecer su validez. Finalmente, los códigos semánticos se correlacionaron con las tres preguntas de investigación mencionadas.

\section{Resultados}

\subsection{Usos}

Agrupamos a continuación los resultados de acuerdo con las preguntas de investigación planteadas. En primer lugar, nos interesa saber cómo acceden y qué hacen los adolescentes en Internet. 
Se destacan varias tendencias respecto de los usos cotidianos, aunque con matices, en función del tipo de grupo de discusión. En primer lugar, se constata la cada vez menor edad de acceso de Internet, que se sitúa en los ocho años en los grupos analizados, junto a las 3 ó 4 horas diarias de conexión que comentan. La mayoría de los participantes de los grupos de discusión tienen al menos un perfil propio en su red social -ficticio o real-, antes de los 14 años, lo que contraviene claramente la legislación española que establece la edad mínima de 14 años para tener un perfil en las redes sociales. Por lo que se refiere a los usos cotidianos en sí, se pueden ordenar comenzando por el acceso a sus redes sociales de preferencia (Tuenti, Facebook), acceso a los programas para hablar y enviar mensajes (Messenger) y la descarga, consumo e intercambio de música y juegos.

El ordenador es omnipresente en las casas y permanece encendido la mayor parte del tiempo.

\subsubsection{Uso del ordenador. Grupo 12-14 años}

- Entrevistador: Eso os iba a decir. ¿Dónde os conectáis?

- Chica: En todos lados. Yo, en la habitación, en el baño si voy a "mear".

- Chico: Uno en mi habitación y otro en el comedor.

- Chica: Yo tengo dos, uno en la habitación de mi hermana y, como tengo WiFi, otro en mi habitación, pero siempre utilizo el suyo, porque es más cerca la WiFi.

- Chico: Yo tengo uno en mi habitación y otro justamente al lado de la habitación de mi hermana. O sea, que son dos de mesa.

En principio no aparece interferencia entre el tiempo de hacer los deberes y el tiempo de ocio. De hecho, Internet es mencionado como herramienta para hacer trabajos del colegio. Por el contrario, no consultan los medios de comunicación y cada vez menos el correo electrónico debido a la publicidad que aparece. Todos tienen teléfono móvil, aunque no acceden a Internet a través de él debido a los costes.

Los participantes en uno de los grupos de discusión confiesan que entre sus conexiones habituales están las páginas pornográficas. En principio, intentan atribuir este comportamiento a los otros compañeros, dando a entender que son conscientes de la reprobación social que conlleva su conducta; sin embargo, acaban admitiendo que entran en sitios pornográficos.

\subsubsection{Uso del ordenador. Grupo 15-17 años}

- Entrev.: Explícanos qué haces por la mañana y qué por las noches.

- Chico: Por la mañana entro en páginas sociales, redes sociales; por la tarde veo páginas guarras y por la noche, si me aburro, también páginas guarras.

Los menores parecen conocer y enfrentar, por un lado, los peligros técnicos asociados al uso de Internet, virus, troyanos y otro tipo de ataques no deseados; mientras que, por otro lado, se muestran muy confiados en sus contactos con cualquier persona no conocida previamente.

- Entrev.: ¿Algún extraño ha entrado en contacto contigo?

- Chica: Si no lo conozco, lo rechazo. 
- Chica: Pues yo no conozco a la mitad de mis contactos en Tuenti. Mucha gente me quiere.

- Chico: Sí, algunas veces, ellos envían invitaciones desde algún lugar, promocionan algún sitio de Internet.

- Chico: Yo sí, pero son gente genial.

Sin embargo, en uno de los grupos de discusión, el "riesgo implícito" de añadir a ciertas personas fue expresado de forma espontánea. Fueron los adolescentes los que explicaron al moderador los riesgos de añadir a un extraño (aunque ellos no usaron este término), sin que el moderador introdujera este asunto o el concepto, lo que va en línea con las observaciones de Patchin and Hinduja (2010). Se ha constatado que existe una percepción de los posibles riesgos de Internet, especialmente en las redes sociales. En este caso, las reacciones mencionadas por los adolescentes para evitarlos fueron desde ignorar las peticiones de extraños en las redes sociales, hasta las confrontaciones verbales con estos extraños.

Paradójicamente, cuando se les pregunta si se consideran débiles en Internet, responden que saben cómo usar el sistema de antivirus. Por el contrario, no mencionan otros tipos de riesgo asociados a las amenazas, presiones o invasión de la privacidad. Es relevante el hecho de que no se utilizara por parte de los integrantes de los grupos de discusión la palabra riesgo, lo que parece indicar que Internet es percibido como un ámbito, en términos generales, seguro.

Afirman colgar fotos en acciones típicas de la vida cotidiana: jugando, cumpleaños, viajes, vacaciones. Y lo hacen con carácter privado. También mencionan las imágenes institucionales colgadas en la página web de un colegio. En sus perfiles no ponen sus fotos aunque, paradójicamente, indican sus datos personales. Uno de los grupos de discusión reconoció que etiquetaban fotos sin previo consentimiento. El barrio en el que viven constituye uno de los datos personales incluidos en sus perfiles, puesto que esperan conocer y agregar a personas de la misma ubicación; esto refuerza su concepción de Internet como lugar seguro.

A lo largo de todos los grupos de discusión se recoge dos usos generales. En primer lugar, se menciona Internet como una herramienta para hacer trabajos y deberes del colegio; y en segundo lugar a través del ciberespacio se accede a contenidos televisivos (principalmente series) constatándose gustos diferenciados por género, en el tipo de series. De hecho, uno de los principales usos en la Red que realizan los menores entrevistados es ver la televisión a la que pueden acceder tanto desde un ordenador como desde una consola. Este dato es importante pues supone la vuelta del papel protagonista de la televisión, si bien de este modo las audiencias virtuales se liberan de la supeditación de la programación. Además, el impacto de los contenidos televisivos se extiende, pues muchas veces los comentarios sobre las series de televisión son los temas principales de conversación que se mantienen en las redes sociales. Otro último dato significativo, en esta misma línea, es que el acceso a contenidos televisivos online reconvierte a la televisión en un instrumento tecnológico con un mayor componente de sociabilidad en el seno de la familia. 


\subsubsection{Uso de la televisión. Grupo 12-14 años}

- Entrev.: Por ejemplo, la tele, ¿en qué puesto está?

- Chica: Después de Internet.

- Chica: Es que también, en Internet, los programas que veo en las cadenas, los veo cuando quiero.

- Chica: Porque por la noche y yo me quedo enseguida. Cuando quiero, me los pongo.

- Chica: Sobre todo las series y películas.

- Entrev.: O sea, que realmente la televisión la consumís a través de Internet.

- Chica: Claro.

Otro aspecto destacado de los grupos de discusión analizados es la diversión. Es preciso tener en consideración que, a pesar de haber codificado estas prácticas de manera independiente en los ocho grupos de discusión, las diferentes prácticas mencionadas participan de la finalidad diversión, puesto que descargar música y compartirla o subir fotos y etiquetarlas, son usos cotidianos pero también tienen como finalidad la diversión; incluso la propia comunicación con amigos a través de chats es una forma de divertirse o de prolongar los momentos de diversión con su círculo de amistad.

Algunos de los participantes en los grupos de discusión demuestran un conocimiento alto de los videojuegos. En este sentido, se detecta un uso mayor por parte de los chicos que de las chicas. Además, se llega a afirmar que, con la edad, son ellos "los que siguen jugando a los videojuegos", asociando este hecho a la idea de que son "más infantiles" y a la consideración de que ellas son "más maduras" o al hecho de que la mayor parte de los juegos están más orientados a una mentalidad masculina. En algún caso, se dice que mientras el hermano está jugando, ella escucha música o habla con sus amigas.

Bajo este mismo código se ha detectado la tendencia de aumentar el consumo de Internet durante el periodo vacacional, sin que por ello se pueda interpretar como un síntoma de adicción.

\subsubsection{Uso de Internet. Grupo 12-14 años}

- Entrev.: ¿Y el fin de semana?

- Chico. Me voy a la calle, saco a los perros por la mañana, me voy hasta las 2 y a las dos como, me duermo y me voy a la calle. [...] pero en verano estoy hasta las cuatro de la mañana conectada.

Por último, los menores entienden por diversión ver vídeos graciosos o series de ficción televisivas que no han podido ver en directo -Física y Química o el Barco-, pero también ven dibujos animados como Dora Exploradora o Phineas y Ferb.

\subsection{Relaciones sociales en Internet. Grupo 15-17 años}

A continuación, nos centramos en los resultados relacionados con los cambios que se han producido, en el caso de que haya sido así, en la visión de los adolescentes sobre las relaciones sociales y emocionales, a consecuencia del uso diario de Internet. 
En las narraciones recogidas se constata que el ciberespacio permite compaginar las relaciones de amistad existentes en el mundo real (Boyd, 2007; Livingstone and Brake, 2010); únicamente para el caso de dos de los miembros del grupo de discusión (15-17 años) se recoge que también utilizaban Skype principalmente para comunicarse con amigos que viven fuera de su comunidad y que, en su mayoría, han conocido en viajes de estudios o en vacaciones.

Otra variedad se encuentra en los menores de origen inmigrante entrevistados, entre los que se detecta de forma minoritaria el uso de las redes sociales para estar conectados con los familiares del país de origen. Por consiguiente, en los grupos analizados se constata la continuidad de las relaciones de amistad en el mundo real traspasadas al espacio virtual, si bien esta constante se acompaña de un nuevo elemento que aparece en escena: un nuevo uso recursivo de la imagen. Nos referimos a la tendencia constatada en los ocho grupos de discusión realizados de colgar fotografías de su vida social en las redes. Pueden llegar a tener de 700 a 3.000 fotografías; y este comportamiento visual puede considerarse como una nueva pauta de socialización.

En este nuevo comportamiento de socialización basado en la imagen, se detectan pautas diferenciadas en función del sexo: las chicas cuelgan más fotos que los chicos. Si bien se recogió esta diferenciación en un único grupo de discusión, es significativo y se establece como dato a verificar en próximos grupos de discusión.

Como ya hemos comentado, en estas nuevas relaciones online, se tiene una actividad muy intensa relacionada con las fotografías. Suelen subir imágenes, verlas, etiquetarlas y comentarlas. En ocasiones, se sienten molestos, no tanto porque supongan un ataque a su intimidad sino porque no "salen bien", en referencia a su aspecto físico.

- Entrev.: ¿Hay un comportamiento distinto? Es decir, vosotros si colgáis muchas fotos y tal por la edad que tenéis.

- Chica: Claro

- Entrev.: Entonces, ¿hay un comportamiento distinto entre los chicos y las chicas?

- Chica: Las chicas somos más de comentar las fotos y hacer las tontas.

- Chico. Sí

- Entrev.: Por eso no queréis admitir a los chicos, ¿no?

- Chica: Claro

- Chica: Que sale uno sin camiseta, pero luego dices, "pero bueno, ¿esto qué es?

- Chica: Luego están las chicas que son como los chicos, que suben fotos sólo agregan a tíos y etiquetan al tío para que vean las fotos.

Todos los participantes en los grupos de discusión han recibido invitaciones por parte de personas a las que no conocían previamente, principalmente por parte de hombres, con la finalidad de comunicar con ellos a través de una red social. Esta práctica afectaba más a las chicas que a los chicos. Reconocen que confían en la información que aparece en los perfiles de los otros usuarios, por lo que, si alguien es desconocido, pero es "amigo de otro amigo", después de un breve control online, esa persona se convierte en un nuevo "amigo" o incluso en una posible cita. 
- Entrev.: ¿Habéis sido objeto de ciber-acoso?

- Chica: En mi caso no fue serio, pero sí.

- Chica: Él me dijo que encendiera la cámara y yo le respondí que ni de broma; pero siguió insistiendo una y otra vez. Al final, lo borré y eso fue el final

- Entrev.: Es decir, que ¿usáis las redes para conocer gente nueva?

- Chico: Claro, por supuesto.

\subsection{Padres $y$ familia}

Por último, nos centramos en la percepción de los adolescentes sobre el papel de los padres y la familia en relación con Internet.

Lo primero que destaca, por lo menos en los pre-adolescentes (12 a 14 años), es que la conectividad se puede considerar como un hecho que se realiza individualmente, mientras que ver la tele tiene un carácter más familiar y social puesto que esta actividad tiene lugar junto a los padres. Algo parecido se ha advertido entre los de mayor edad: es habitual que en cada familia haya más de un ordenador, incluso en determinadas ocasiones cada miembro de una familia tiene un ordenador, ya sea de consola ya sea portátil. En último extremo, resulta relevante que cada individuo/a pueda llegar a tener un ordenador y, por el contrario, solo exista una televisión que se ve de forma familiar, a través de la que se establecen límites en los contenidos considerados no apropiados. Mientras que en la Red este control disminuye.

Los menores, entre 12 y 14 años, no muestran las mismas ideas sobre el conocimiento de sus padres acerca de la tecnología en general y sobre Internet en particular, en comparación con su conocimiento. Algunos afirman que es mayor y otros, que es menor. En cualquier caso, muchos de ellos sostienen que sus padres no manejan ni conocen bien Internet. De hecho, ponen las contraseñas ellos mismos para impedir a sus mayores el control de los contenidos; si bien hay padres que tienen sus propios perfiles en las redes sociales.

En el ámbito de los adolescentes situados entre los 15 y los 17 años, no encontramos una idea general a este respecto. Algunos de los participantes señalan que sus padres están inmersos en las redes sociales o en otras actividades (como puede ser la compra online), otros tienen una vida online limitada y, finalmente, otros ni siquiera utilizan el ordenador. Esto tiene importancia, habida cuenta de que son éstos últimos quienes se implican más en las tareas de control y seguridad. En los grupos de discusión vuelve a aparecer otra figura clave: la del hermano o hermanos, que muestran una clara vinculación con actividades online. De igual modo, afirman que el primer contacto con Internet requiere de la compañía de los padres.

\subsubsection{Padres y familia. Grupo 15-17 años}

- Chica: Y aprendemos solos. Para eso no hay un manual o algo.

- Entrev.: O sea, que creéis que realmente es bueno, por ejemplo, empezar más o menos joven a Internet porque así aprendes cosas.

- Chico: Sí.

- Chico: Sí, claro que sí, pero escúchame: eso lo puede ver siempre y cuando tenga a una persona mayor para ver cómo lo hace, ¿sabes? En qué se mete, porque si no... 
No se detecta ninguna diferencia clara entre las familias tradicionales y las monoparentales en el control o comportamiento digital en esta franja de edad. Además, el acceso (también temprano, desde los diez y once años) a Internet, siempre se realiza, según los participantes, con el consentimiento paterno y materno. Es normal encontrarse con menores que son responsables y que no se aventuran a acceder a páginas inapropiadas. Además creen que si cometieran alguna imprudencia se acabaría sabiendo. La relación familiar es la típica de la edad en que se encuentran los participantes: rebeldía y contestación hacia los padres. Sólo se acude a los padres en caso de problema grave.

- Entrev.: Tenéis ¿Tenéis, por ejemplo, más o menos claro que si os pasa alguna cosa un poco más fuerte, acudiríais a vuestros padres?

- Chica: No, para nada.

- Chico: No.

- Chica: A mí me pasaron cosas peores y no he ido.

- Chica: A mí me pasó, en el Metroflog, que antes lo usaba mucha gente, y subieron una foto mía en un Metroflog que no conocía, o sea, no sabía de quién era. De repente subieron una foto mía y empezaron a comentar de mí diciendo un montón de cosas. Era anónimo, claro. Y yo, claro, se lo dije a mi madre. Digo. "mamá, mira lo que me ha pasado" y mi madre decía. "ya te enterarás de quién será". Es más, ella dijo. "voy a buscar a un informático para que sepas quién ha sido y lo denunciamos" y no sé qué. Yo digo. "mamá, si eso yo voy al instituto, que seguro que ha sido alguna del instituto". Llegué y al día siguiente lo sabía ya. Le dije. "tú, pon eso y ten cuidado con lo que te puede pasar". O sea, la amenacé y lo quitó al día siguiente.

\section{Conclusiones}

Los resultados obtenidos en este trabajo son fruto de un proyecto estatal que se acercó a conocer los usos y prácticas de los adolescentes en Internet. La opción cualitativa de este trabajo ha permitido adentrarse en las percepciones de los menores y adolescentes, conforme a sus narrativas y discursos. Esto ha facilitado confirmar algunas tendencias destacadas en la bibliografía tanto en España como a nivel internacional, destacar nuevo hallazgos, así como plantear algunas líneas de futuro para seguir estudiando los significados de los usos de los nativos digitales.

En primer lugar, destaca la disminución progresiva de la edad a la que los menores se empiezan a conectarse a Internet, así como el asentamiento de la multitarea como característica genérica en el colectivo analizado. Parece, por tanto, obvio que la limitación establecida en la legislación española a los 14 años para tener perfil propio en las redes sociales, ha quedado sobrepasada por la realidad, entre otras razones, porque la pertenencia a una red social es vivida por los participantes en los grupos de discusión como criterio de inclusión social.

Asimismo, se confirman los mecanismos de mediación familiar, donde el peso básico de influencia recae en los hermanos, así como la individualización de la experiencia digital: la soledad del propio cuarto y, al mismo tiempo, estar y quedar en grupo mientras cada uno está pendiente de su móvil. Directamente relacionado con esto, se aprecia algo que algunos estudios ya habían perfilado: la combinación de la 
vida y los espacios online y offline, cuya plataforma por excelencia son las redes sociales, en tanto que entramado para sus relaciones sociales.

No se pueden establecer tendencias generales sobre cómo son actualmente las dinámicas en las relaciones afectivas. Por el contrario, las narraciones analizadas nos permiten detenernos y adentrarnos en microrealidades concretas que sirven para expresar el dinamismo comunicativo de los escenarios virtuales, que para los menores y adolescentes participantes están cargados de seguridad. Se observa cómo el placer de la amistad se adapta al tiempo y consumo del siglo XXI, para dar lugar a nueva intimidad digital. Otros autores -Fernández Porta (2010)- aventuraron que las emociones son un producto para el mercado generado por Facebook y Twitter, medios que dominan y comercializan los sentimientos en la red y que han redefinido la noción de intimidad y privacidad.

Es significativa la tendencia detectada en relación con la intensa actividad de subir fotografías, etiquetarlas y comentarlas. Como hemos visto, en las ocasiones en que los participantes se sienten contrariados, la razón no proviene tanto de que perciban invadida su intimidad cuando algún amigo cuelga fotos de grupo o etiquete determinada foto, sino porque no se ven bien representados en ellas. Las redes sociales aparecen en este sentido como el lugar de nuevas prácticas de espectacularización corpórea: hiperexposición de fotografías y resignificación identitaria desde alusiones estéticas.

El análisis de los relatos de los adolescentes ha permitido observar una tendencia relevante que tiene que ver con la consideración e interpretación del término riesgo. $\mathrm{Su}$ visión difiere claramente de la comprensión existente en el marco familiar o educativo. Agregar y quedar con personas desconocidas, ofrecer datos privados, colgar y comentar fotos ajenas son conductas generalizadas en los grupos analizados, así como la reformulación de otras prácticas vinculadas a la privacidad apuntan a una diferente concepción del riesgo e invitan a profundizar en las características de esta cuestión en estudios futuros.

De la misma forma, algunas de las prácticas apuntadas por los adolescentes en este trabajo como la conexión cada vez más temprana, el creciente número de horas de navegación, así como la tendencia -en los chicos- a conectarse constantemente a los juegos y el acceso a páginas no recomendadas, constituyen acciones que en algunos casos podrían ser englobadas en la denominación de ciberpatología. Esto abriría nuevos cauces de investigación orientados al tratamiento de patologías acentuadas con Internet, en especial adicciones a juegos y trastornos alimentarios. En este sentido, conviene estar atento desde el momento mismo de su aparición contrastando con los relatos en primera persona, lo que ayudaría a una comprensión de las ciberpatologías desde una dimensión social y cultural.

\section{Referencias bibliográficas}

BARKHUUS, Louise \& TASHIRO, Juliana (2010): "Student socialization in the age of Facebook". Proceedings of the 28th Annual ACM Special Interest Group on Computer on Computer Human Interaction Conference on Human Factors in Computing Systems (SIGCHI '10). New York, ACM Press, pp. 133-142. 
BOYD, Danah (2007): "Why youth heart social network sites: the role of networked publics in teenage social life". In BUCKINGHAM, David (Ed.): Youth, identity, and digital media. Cambridge. MA, MIT Press, pp. 119-142.

CHEUNG, Christy; CHIU, Pui-Yee; \& LEE, Matthew (2011): "Online social networks: Why do students use Facebook?" In Computers in Human Behavior, 27, pp. 1337-1343.

DUERAGER, Andrea \& LIVINGSTONE, Sonia (2012): How can parents support children's internet safety? EU Kids Online, London, UK: http://www2.lse.ac.uk /media@1se/research/EUKidsOnline/EU\%20Kids\%20III/Reports/ParentalMediation.pdf [Fecha de consulta: 18 de julio de 2012].

ELLISON, Nicole; STEINFIELD, Charles; \& LAMPE, Cliff (2007): "The benefits of Facebook friends: Social capital and college students' use of online social network sites" In Journal of Computer Mediated Communication, 12, pp. 1143-1168.

FERNÁNDEZ, Eloy (2010): Eros. La superproducción de los afectos. Madrid, Anagrama.

FOUCAULT, Welles \& MARKOV, August (2009): “Teens and communication technology: The co-construction of privacy and friendship in mediated communication". Proceedings of the International Communication Association 2009 Annual Meeting (ICA '09) Chicago, IL, pp. 1-27.

GARCÍA, Antonio; SENDÍN, José Carlos; CATALINA, Beatriz (2012): "Usos de Internet y de redes sociales entre los adolescentes en España”. Sphera Pública, núm. 12. pp. 83-100.

GARCÍA JIMÉNEZ, Antonio (coord., 2010): Comunicación y comportamiento en el ciberespacio. Actitudes y riesgos de los adolescentes. Barcelona, Icaria.

GARMENDIA, Maialen; GARITAONANDIA, Carmelo et al. (2011): "Riesgos y seguridad en Internet: los menores españoles en el contexto europeo". En www.ehu.es/eukidsonline. [Fecha de consulta: 12 de marzo de 2012].

GROSS, Elisheva (2004): “Adolescent Internet use: What we expect, what teens report”. Journal of Applied Developmental Psychology, 25 (6), pp. 633-649.

INSTITUTO NACIONAL DE ESTADÍSTICA (INE) (2012): Encuesta de hogares sobre equipamiento y uso de Tecnologías de la Información y la Comunicación. Base de datos INE base, http://www.ine.es [Fecha de consulta: 12 de enero de 2013].

ITO, Mizuko; BAUMER, Sonja; BITTANTI, Matteo; BOYD, Danah; CODY, Rachel; HERR-STEPHERSON, Becky et al. (2009): Hanging out, messing around, and geeking out: Kids living and learning with new media. Cambridge, MA, The MIT Press.

LAM, Lawrence; PENG, Zi-Wen; MAI, Jin-Cheng; \& JING, Jin (2009): "Factors associated with Internet addiction among adolescents". CyberPsychology and Behavior, 2009, 12 (5), pp.551-555. http://www.ncbi.nlm.nih.gov/pubmed/19619039. [Fecha de consulta: 14 de septiembre de 2012]. 
LENHART, Amanda \& MADDEN, Mary (2007): "Social networking websites and teens: An overview": http://www.pewinternet.org/pdfs/PIP_SNS_data_Memo _Jan_2007.pdf [Fecha de consulta: 18 de febrero de 2012].

LENHART, Amanda; PURCELL, Kristen; SMITH, Aaron \& ZICKUHR, Kathryn (2010): "Social media and young adults". In http://pewinternet.org/Reports/2010/Social-Media-and-Young-Adults.aspx [Fecha de consulta: 18 de febrero de 2012]

LIU, Quin-Xue et al. (2012): "Parent-adolescent communication, parental Internet use and Internet-specific norms and pathological Internet use among Chinese adolescents". Computers in Human Behavior, 28, pp. 1269-1275.

LIVINGSTONE, Sonia (2008): “Taking risky opportunities in youthful content creation: Teenagers' use of social networking sites for intimacy, privacy and self-expression". New Media \& Society, 10, pp. 393-411.

LIVINGSTONE, Sonia \& HELSPER, Ellen (2008): "Parental Mediation of Children's Internet Use". Journal of Broadcasting \& Electronic Media, 52 (4), pp. 581-599.

LIVINGSTONE, Sonia \& BRAKE, David (2010): "On the rapid rise of social networking sites: New findings and policy implications". Children \& Society, 24, pp. 75-83.

LIVINGSTONE, Sonia; HADDON, Leslie; GÖRZIG, Anke; \& ÓLAFSSON, Kjar$\tan$ (2011): "Risks and safety on the internet: The perspective of European children". In: http://eprints.lse.ac.uk/33731/ [Fecha de consulta: 18 de marzo de 2012].

MESCH, Gustavo (2001): "Social relationships and Internet use among adolescents in Israel". Social Science Quarterly, 82, pp. 329-340.

MESCH, Gustavo \& TALMUD, Ilan (2007): "Similarity and the quality of online and offline social relationships among adolescents in Israel". Journal of Research on Adolescents, 17, pp. 455-466.

NIE, Nicholas (2001): "Stability, interpersonal relationships and the Internet: Reconciling conflicting findings". American Behavioral Scientist, 45, pp. 420-435.

OCHAITA, Eesperanza; ESPINOSA, Ma Ángeles; y GUTIÉRREZ, Héctor (2011): "Las necesidades adolescentes y las nuevas tecnologías de la información y la comunicación". Revista de Estudios de Juventud, 92, pp: 87-110. En línea: http://www.injuve.es/contenidos.downloadatt.action?id=686434135 [Fecha de consulta: 12 de septiembre de 2011].

PARK, Soo Kyung; KIM, Jae Yop \& CHO, Choon Bum (2008): "Prevalence of Internet Addiction and correlations with family factors among South Korean adolescents". Adolescence, 43 (172), pp. 895-909.

PURCELL, Kristen (2011): “Trends in teen communication and social media use" (Pew Internet \& American Life Project). Presentation given at Joint Girl Scout Research Institute/Pew Internet Webinar: http://www.pewinternet.org/Presentations/2011 /Feb/PIP-Girl-Scout-Webinar.aspx. [Fecha de consulta: 25 de mayo de 2012]. 
PATCHIN, Justin \& HINDUJA, Sameer (2010): "Trends in online social networking: Adolescent use of MySpace over time". New Media \& Society, 12 (2), pp. 197216.

RÍO, Javier; SÁDABA, Charo; y BRINGUÉ, Xavier (2010): 'Menores y redes ¿sociales?: De la amistad al cyberbullying'. Revista de Estudios de Juventud, (88), pp. 115-129. En línea: http://www.injuve.es/contenidos.downloadatt.action ?id=1880411574. [Fecha de consulta: 19 de septiembre de 2011].

SÁNCHEZ BURÓN, Adolfo y FERNÁNDEZ MARÍN, María Poveda (2010): Generación 2.0, 2010. Hábitos de los adolescentes en el uso de las redes sociales. Madrid, Universidad Camilo José Cela. En: http://www.slideshare.net/ucjc/generacin-20-hbitos-de-los-ad. [Fecha de consulta: 12 de septiembre de 2011].

SÁNCHEZ NAVARRO, Jordi y ARANDA, Daniel (2011): "Internet como fuente de información para la vida cotidiana de los jóvenes españoles". El Profesional de la Información, 20 (1). En: http://www.elprofesionaldelainformacion.com/contenidos/2011/enero/04.html [Fecha de consulta: 13 de septiembre de 2011].

SUBRAHMANYAM, Kaveri; REICH, Stephani; WAECHTER, Natalia \& ESPINOZA, Guadalupe (2008): "Online and offline social networks: Use of social networking sites by emerging adults". Journal of Applied Developmental Psychology, 29, pp. 420-433.

SUBRAHMANYAM, Kaveri \& SMAHEL, David (2011): Digital Youth: The Role of Media in Development. New York, Springer.

VALCKE, Martin et al. (2011): "Long-term study of safe Internet use of young children". Computers \& Education, 57, pp. 1292-1305.

VALKENBURG, Pati \& PETER, Jochen (2009): "Social consequences of the Internet for adolescents: a decade of research". Current Directions in Psychological Science, 18 (1), pp. 1-5.

VALKENBURG, Pati \& PETER, Jochen (2011): “Online communication among adolescents: an integrated model of its attraction, opportunities, and risks". Journal of Adolescent Health, 48(2), pp. 121-127.

VIÑAS, Ferrán; JUAN, Jaume; VILLAR, Esperanza; CAPARROS, Beatriz; PÉREZ, Isabel y CORNELLA, Montserrat (2002): "Internet y psicopatología: las nuevas formas de comunicación y su relación con diferentes índices de psicopatología". Clínica y Salud, 13, pp. 235-256. http://redalyc.uaemex.mx/redalyc/pdf /1806/180618090001.pdf [Fecha de consulta: 23 de agosto de 2012].

WALSH, Shari; WHITE, Katherine \& YOUNG, Ross (2009): "The phone connection: A qualitative exploration of how belongingness and social identification relate to mobile phone use amongst Australian youth". Journal of Community \& Applied Social Psychology, 19, pp. pp. 225-240.

YEN, Ju-Yu; YEN, Cheng-Fang; CHEN, Cheng-Chung; CHEN, Sue-Huei; \& KO, Chih-Hung (2007): "Family Factors of Internet Addiction and Substance Use Ex- 
perience in Taiwanese Adolescents". CyberPsychology \& Behavior; 10 (3), pp. 323-329. 